\title{
The Spectral Classification of Stars over the last 200, 100, 75 years and in the future
}

\author{
Christopher J. Corbally ${ }^{1}$ and Richard O. Gray ${ }^{2}$ \\ ${ }^{1}$ Vatican Observatory, University of Arizona, \\ Tucson, AZ 85721, USA \\ email: corbally@as.arizona.edu \\ ${ }^{2}$ Dept. of Physics \& Astronomy, Appalachian State University, \\ Boone, NC 28608, USA \\ email: grayro@appstate.edu
}

\begin{abstract}
This year 2018 has great historical and current significance for stellar spectral classification. Two hundred years ago in Reggio Emilia, Italy, was born Angelo Secchi, a pioneer of observing and classifying the spectra of stars. At the beginning of the IAU, almost a hundred years ago, one of its original Commissions was entitled the Spectral Classification of Stars, from which was generated Commission 45, Spectral Classification and Multi-band Colour Indices. And seventy-five years ago, was published the system-changing MKK, An Atlas of Stellar Spectra. Through this necessarily brief, historical view we shall recall how spectral classification, supported internationally by the IAU, continually updated its techniques, while remaining anchored to standards. This has ensured that the MK classification process stays very relevant to the initial characterizing of stars in the 21st century era of large spectral surveys.
\end{abstract}

Keywords. History and philosophy of astronomy, techniques: spectroscopic, stars: fundamental parameters, stars: atmospheres, stars: evolution, the Galaxy: stellar content, galaxies: abundances, galaxies: stellar content

\section{From 200 years ago, the pioneers}

To claim two hundred years' worth of spectral classification is stretching a point, but it is certainly true that Angelo Secchi was born on 28 June 1818 in Reggio Emilia, Italy. And four years earlier Joseph von Fraunhofer in Munich had first observed solar and stellar spectra, naming spectral features even used today. So, the history of at least stellar spectroscopy goes back a good century before the IAU was founded.

Gustav Kirchhoff in 1860 started a real revolution by interpreting the dark lines that Fraunhofer had seen in the spectrum of the Sun in terms of the glowing chemical gases that Kirchhoff was producing in his laboratory. This work stimulated observations of other stellar spectra by Giovanni Battista Donati in Florence, William Huggins in London, Lewis Morris Rutherfurd in New York, Angelo Secchi in Rome, and some three others. Huggins and Secchi could both see that the elements they knew on Earth existed in the stars, and Secchi even surmised that the breadth of hydrogen lines in Sirius indicated a great pressure in its atmosphere. The change of what was up until then, positional astronomy, into modern astrophysics had begun.

Classification of new phenomena is basic to science, so while Donati made modest descriptions of spectra, Rutherfurd amassed sufficient of his own to classify them into three groups, corresponding to what we now call the late-type stars, the late-B to early-F stars, and the OB stars. However, the most prolific observer and classifier was Secchi. 


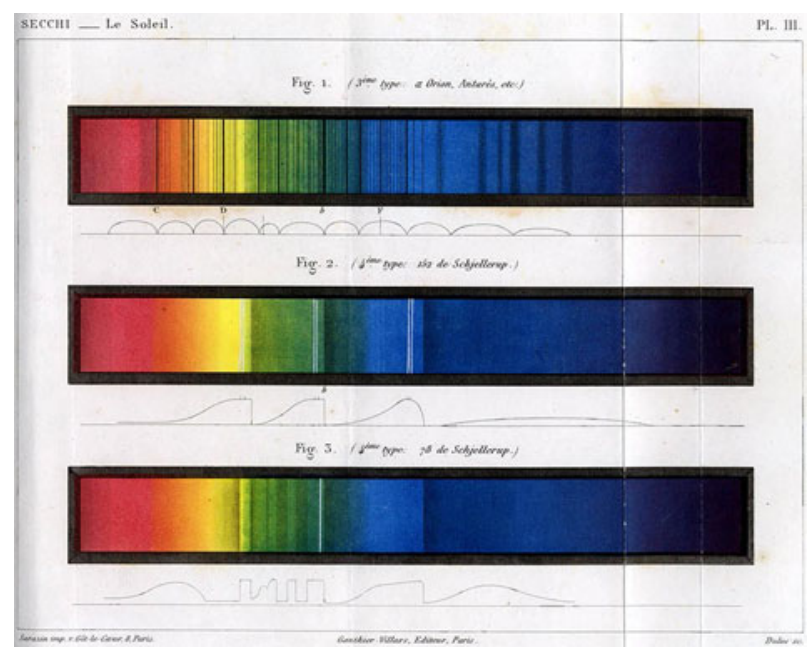

Figure 1. Secchi's examples in Le Soleil of an M supergiant (top) and two carbon stars.

His detailed drawing of the spectra of some 4000 stars enabled him to sort them into a limited number of distinct types, even recognizing carbon in his type IV (Fig. 1).

This pioneering in stellar spectroscopy and classification continued in the latter part of the nineteenth century. Unfortunately, Norman Lockyer in London put the cart before the horse in letting his theory on the evolution of stars dictate his classification scheme, but at least he helped distinguish giants and supergiants from dwarf stars. When classifiers let the spectra group themselves naturally, then a consistent ordering of types began to appear, modelled on the Secchi ones. Hermann Carl Vogel, of Bothkamp then Potsdam, was one of those who started with the Secchi types, and he later modified his classification scheme upon the discovery of helium. Around this late-nineteenth century period, a very significant scheme began to emerge, thanks to Edward Pickering and Williamina Fleming, and it was enshrined in the 1890 Draper Memorial Catalogue of stars. (Henry Draper's wife, Mary Anna Draper, wanted to memorialise her husband who was an amateur and had started photography of objective-prism spectra, so establishing the method used in the Catalogue.) Antonia Maury introduced her own modifications into this Harvard scheme, and later through Annie Jump Cannon we find the familiar sequence-OBAFGKM - appearing for the first time.

\section{From 100 years ago, the IAU era}

The last of the four Draper Catalogues was published in 1912, but by then a decision was clearly needed over which of the several prevailing spectral classification schemes was going to be the most useful. Here the newly-formed IAU made a timely entrance: the tentative adoption of the Draper system by a Standing Committee on the Classification of Stellar Spectra became formal in Rome at the first General Assembly of the IAU in 1922 .

That first General Assembly also constituted Commission 29, Spectral Classification of Stars. Its first president was W.S. Adams of Pasadena, and its members (A.J. Cannon, R.H. Curtiss, A. Fowler, A. de Gramont, M. Hamy, H.F. Newall, J.S. Plaskett, H.N. Russell) are all very much part of the history of stellar spectroscopy. In its early years classification dominated the discussions of members, but a change of the commission's name to Stellar Spectra in 1935 under president H.N. Russell showed that the physical interpretation of spectra naturally followed the technical problem of classification. 
Some 15 years later, Otto Struve reflected on that relationship: "But while many persons engaged in spectral classification could probably get along quite well without the astrophysical interpretations, the astrophysicist cannot get along without the help of his colleague who is interested in classification" (Struve 1950). This wisdom may well have resulted from Stuve's regular contact with W.W. Morgan, also working at Yerkes Observatory. It was becoming clear that spectral classification had two goals: to locate a star in the context of the broad population of stars, e.g., its location in the HR diagram; and to identify peculiar and astrophysically interesting stars.

Without too much surprise it was during the next General Assembly that Commission 45, Spectral Classification and Multi-band Colour Indices, arose as a commission distinct from Commission 29 and with W.P. Bidelman as its first president. However, as Stuve would expect, over the next 50 years these two sister commissions very much collaborated in meetings, etc., to the overall benefit of stellar astrophysics. In 1979 Commission 45 changed its name to just Stellar Classification, so including photometric classification in its work. But we have rather jumped ahead in the progress of classification.

\section{From 75 years ago, the MK era}

The year 1943 was perhaps the most significant over the last 200 for spectral classification. It saw the publication of An Atlas of Stellar Spectra (Morgan, Keenan \& Kellman 1943), which established the MK System of classification. The power of this new system lay in it being anchored to a comprehensive grid of standards in spectral type and luminosity class, and these standards do not change. So, an 'MK classification' is a description of how the spectrum of a star appears at medium resolution in the blue-green wavelength region and not a listing of its physical parameters. Even when new improved values for the surface temperature, pressure, and composition emerge from observations and theory, the Sun still remains a "G2 V" class star. As such it, and fellow early-G dwarf stars, can be compared among themselves with respect to their temperatures, pressures, and even compositions. This reminds us that in addition to spectral type and luminosity class, a third dimension was also recognized in the MK System, and this dimension, one that includes element abundances and peculiar stars, has been under refinement ever since.

Any illustration of the power of the MK System must include the 1957 Vatican workshop on Stellar Populations (O'Connell 1958). Significant contributions to the conference's successful distinction of five galactic populations came from Morgan's proof of spiral arms in the Galaxy and his comparison of integrated galactic spectra, together with the development by Nancy Roman of a "third parameter, the division by kinematical properties, as shown by spectral peculiarities", denoting "presumably old population I and mild II stars". Considerable work on stellar populations followed the Vatican conference using objective-prism spectroscopy and wide-field telescopes.

Among other significant conferences on the MK System have been: the 1977 IAU Colloquium 47, Classification of the Future, in the Vatican City and dedicated to Angelo Secchi; the 1983 workshop in Toronto, The MK Process and Stellar Classification, which both Keenan and Morgan were able to attend; and the 1993 meeting in Tucson, Arizona, The MK Process at 50 Years: A Powerful Tool for Astrophysical Insight.

In the 1988 IAU Transactions report, the then-president, R.F. Garrison, made a remark that stellar classification provides "systems" for comparison with new types of stars. This was borne out in the next decade or so by the discovery and definition of the L, T and Y classes of ultra-cool dwarfs (Fig. 2). The exciting development regarding L and T dwarfs was discussed in a C45 session during the XXIV IAU GA in Manchester in 2000. Research on these classes expanded rapidly so that by 2006 the T-dwarf standards became unified. In 2012 R.O. Gray's report recorded that Y dwarfs had been discovered as a class distinct from $\mathrm{T}$ dwarfs, and that low-gravity L0-L5 dwarfs had been defined. Developments in 


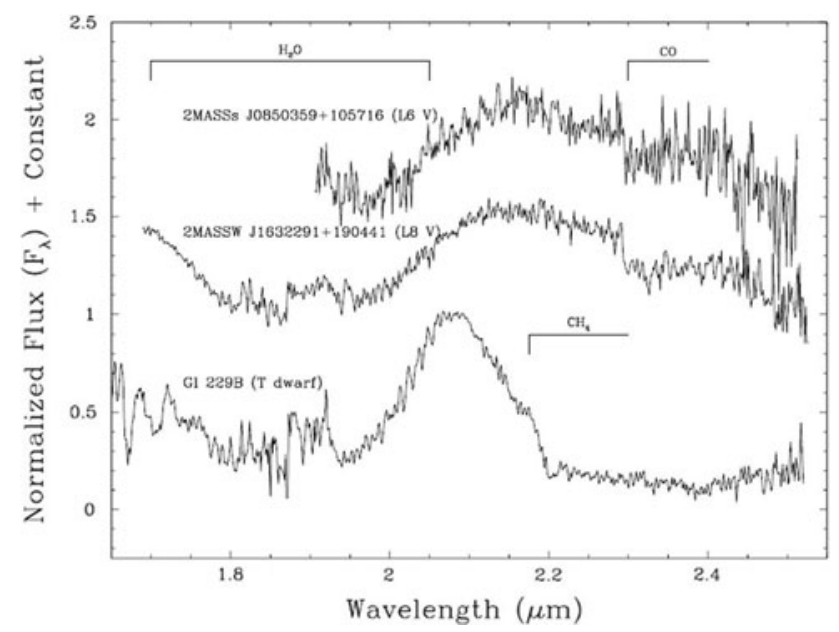

Figure 2. K-band spectra of two L dwarfs and the prototype T dwarf (bottom). (Figure from Kirkpatrick et al. 1999)
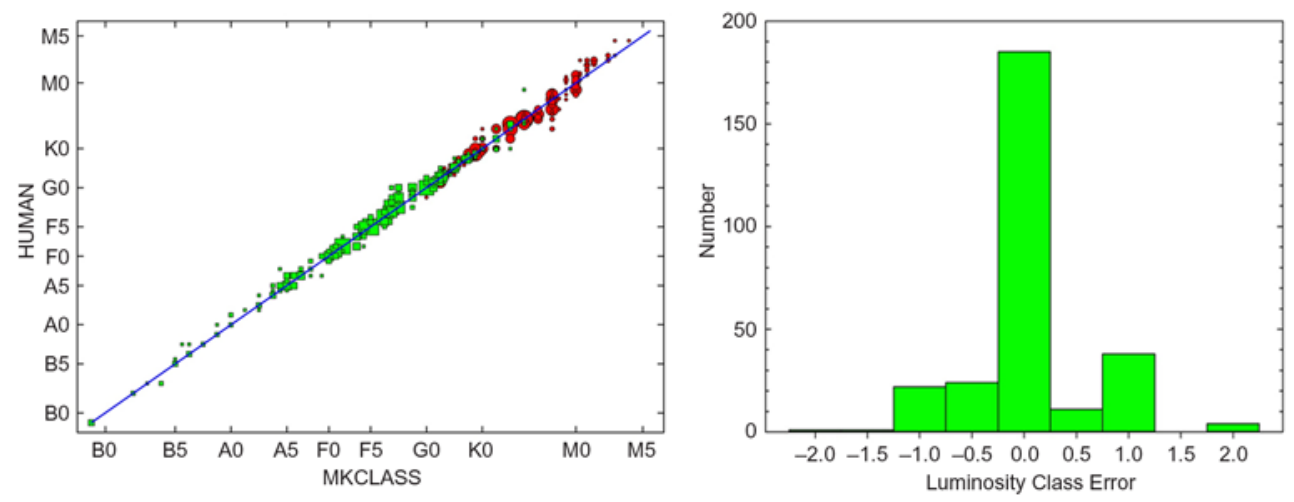

Figure 3. Comparisons between human classifiers and MKCLASS for spectral type (left), and for luminosity (the right-hand histogram of difference between human and MKCLASS).

near-IR spectral surveys were crucial to these discoveries. The traditional blue-green region for the classification of spectra was being extended into the infrared.

\section{From the present into the future}

Secchi, who had visually observed and hand-drawn his spectra, and the others mentioned in this history, would be thrilled by what the digital era has brought to the power of spectral classification. The succession of digital detectors, by their steady increase in sensitivity, size and wavelength region, have made the observation of very faint objects, like brown dwarfs, possible. Multiple fibre spectrographs have brought the same sensitivity to wide-field surveys. Coupled with deep photometric surveys like PanStarrs, LSST, Gaia and soon WFIRST, multi-object spectroscopy opens up new possibilities for the study of Galactic structure and populations, clusters, star-forming regions and searches for specially interesting stars.

To cope with all these spectra, the MKCLASS code, an expert system, was developed so that computers can classify spectra in the same way humans do: via direct comparison with the MK Standards. Its success is clear when compared with human classifiers (Fig. 3). 
It has been used to classify spectra from the 4000-fibre LAMOST telescope for fields covering the Kepler space telescope area (Gray et al. 2016). Interesting stars discovered in this database by MKCLASS include carbon-peculiar and chemically-peculiar red giants, barium dwarfs (s-process enhanced), magnetically active stars, Ap stars and the still enigmatic lambda Boötis stars, which featured 75 years ago in the MKK Atlas. Old problems can be revisited through stellar spectral classification and developing techniques, while new questions become uncovered.

\section{Discussion}

Hernandez: Not mentioned was Henry Draper as one of the pioneers of stellar spectroscopy, as he was the first to capture a spectrum on a photographic plate. Nor was the research which followed, based on this achievement.

Corbally: There were so many interesting things that had to be left out of my short talk, but you are right about the importance of Henry Draper's pioneering spectroscopy, which led, via his wife, to the catalogue in his name. I shall include the mention you suggest in the written version of my talk.

\section{References}

Gray, R. O., Corbally, C. J., De Cat, P., Fu, J. N., Ren, A. B., Shi, J. R., Luo, A. L., Zhang, H. T., Wu, Y., Cao, Z., Li, G., Zhang, Y., Hou, Y., \& Wang, Y. 2016, AJ 151, 13

Kirkpatrick, J. D., Reid, N. I., Liebert J., Cutri, R. M., Nelson, B., Beichman, C. A., Dahn, C. C., \& Monet, D. G. 1999, ApJ 519, 802

Morgan, W. W., Keenan, P. C., \& Kellman, E. 1943, An Atlas of Stellar Spectra. Chicago: University of Chicago Press

O'Connell, D. J. ed. 1958, Stellar Populations. Amsterdam: North Holland Publishing Co.

Struve, M. O. 1950, in Transactions of the IAU VII, 289 